\title{
Structure-guided engineering of a Thermobifida fusca cutinase for enhanced hydrolysis on natural polyester substrate
}

Qilei Dong ${ }^{1,3+}$, Shuguang Yuan ${ }^{4 \dagger}$, Lian $\mathrm{Wu}^{1 \dagger}$, Lingqia $\mathrm{Su}^{5}$, Qiaoling $\mathrm{ZhaO}^{1}$, Jing $\mathrm{Wu}^{5}$, Weixue Huang ${ }^{1 *}$ and Jiahai Zhou ${ }^{1,2^{*}}$ (D)

\begin{abstract}
Cutinases could degrade insoluble polyester, including natural cutin and synthetic plastic. However, their turnover efficiency for polyester remains too low for industrial application. Herein, we report the 1.54-Å resolution X-ray crystal structure of a cutinase from Thermobifida fusca and modeling structure in complex with a cutin mimic oligo-polyester $\mathrm{C}_{24} \mathrm{H}_{42} \mathrm{O}_{8}$. These efforts subsequently guided our design of cutinase variants with less bulky residues in the vicinity of the substrate binding site. The L90A and I213A variants exhibit increased hydrolysis activity (5- and 2.4-fold, respectively) toward cutin and also showed enhanced cotton scouring efficiency compared with the wild-type enzyme.
\end{abstract}

Keywords: Cutinase, Polyester cutin, Enzyme engineering, Cotton scouring, Loop flexibility

\section{Introduction}

Cutin, a natural polyester, forms a three-dimensional network structure of protective cuticle in higher plants, in which waxy material and pectin are embedded (Fig. 1a) (Kolattukudy 2001). Cotton scouring which removes the hydrophobic cuticle of raw cotton is an essential treatment to increase cotton wettability and dyeability. Conventionally, scouring is carried out in the hot $\mathrm{NaOH}$ aqueous solution. However, such non-specific alkaline scouring generates environmental pollutants and decreases fabric tension. Alternatively, an enzymatic technique might be applied as a more eco-friendly technique, producing fabrics with excellent tension because of the specific removal of non-cellulose materials (Wang et al. 2006).

\footnotetext{
*Correspondence: wxhuang@sioc.ac.cn; jiahai@sioc.ac.cn

${ }^{\dagger}$ Qilei Dong, Shuguang Yuan and Lian Wu contributed equally to this work

1 State Key Laboratory of Bioorganic and Natural Products Chemistry, Center for Excellence in Molecular Synthesis, Shanghai Institute of Organic Chemistry, Chinese Academy of Sciences, 345 Lingling Road, Shanghai 200032, China

Full list of author information is available at the end of the article
}

Cutinases that could degrade the protective cutin of plants are commonly secreted by plant pathogens. The fungal Fusarium solani pisi cutinase was the first cutinase to be functionally characterized (Purdy and Kolattukudy 1973, 1975). Cutinases belong to the serine hydrolase superfamily, and catalyze the hydrolysis of ester bond. These enzymes have broad substrate ranges, including soluble fatty acids, triglycerides, and insoluble polyesters like cutin, polyethylene terephthalate (PET), highlighting their potentials for use in industrial applications such as bioscouring and the degradation and/or synthesis of plastics (Nyyssola 2015; Liu et al. 2009).

A cutinase from the thermophilic bacterium Thermobifida fusca was identified, and it showed superiority over fungal cutinases in thermostability, $\mathrm{pH}$ tolerance, and resistance to surfactants and organic solvents (Chen et al. 2008; Hegde and Dasu 2014). However, enzymatic assays revealed that T. fusca cutinase prefers short chain esters as substrates and has lower turnover rate for polyesters (Chen et al. 2010a). Many studies attempt to relate the substrate preferences of cutinases to their structural characteristics, with the aim of engineering biocatalysts with desired 


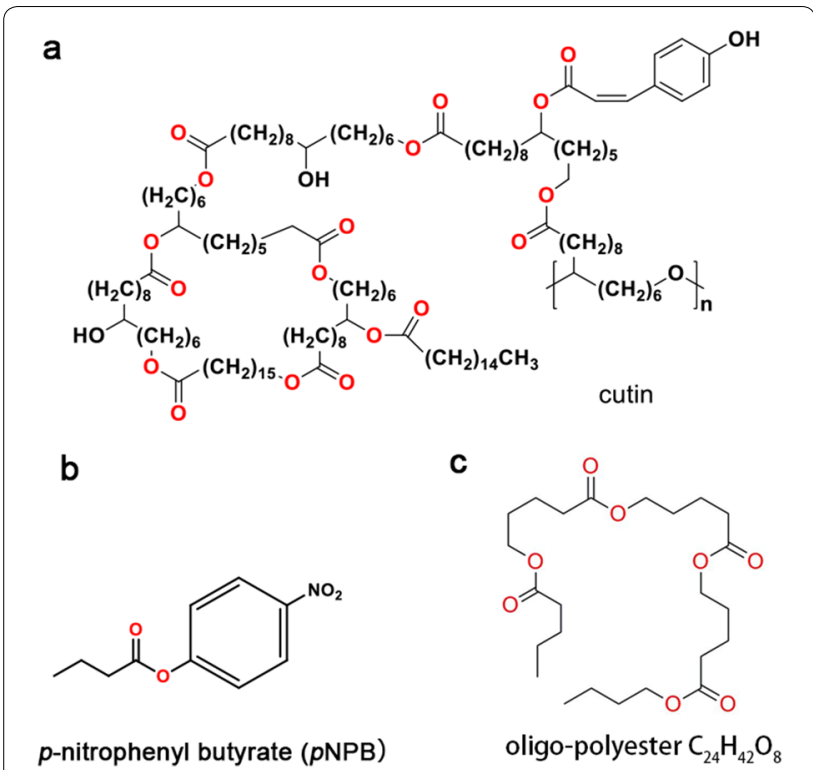

Fig. 1 Structures of compounds used in this study. a Cutin is the natural polyester substrate of cutinase. $\mathbf{b} p N P B$ is a small-model substrate of cutinase. The intramolecular ester oxygen atoms are colored in red. c The oligo-polyester $\mathrm{C}_{24} \mathrm{H}_{42} \mathrm{O}_{8}$ was used for molecular docking experiments properties (Araujo et al. 2007; Ribitsch et al. 2011, 2017; Han et al. 2017; Joo et al. 2018; Kitadokoro et al. 2012; Oda et al. 2018; Silva et al. 2011; Tournier et al. 2020). Extensive experimental evidence has demonstrated that the accessibility of the enzyme's active site is the major factor enabling the biodegradability of polymers (Fecker et al. 2018; Saavedra et al. 2018). Several engineering efforts have successfully exploited the enlargement of the active sites of enzymes to increase catalytic activity on synthetic aromatic polyesters (Austin et al. 2018; Han et al. 2017; Joo et al. 2018). Nevertheless, the exact mechanism through which cutinases recognize the natural, net-shaped aliphatic polyester cutin remains elusive. A detailed understanding of the substrate-enzyme interactions could guide the enzyme engineering to obtain variants with enhanced catalytic efficiency. Herein, based on the protein-substrate information from our $T$. fusca cutinase structure, we designed and successfully obtained enzyme variants with increased hydrolysis activity towards cutin and enhanced cotton scouring performance. Fundamentally, our work highlights that protein engineering could notice functional contributions from residues in the vicinity of substrate binding site which might confer indirect effects on desired catalytic activity.

\section{Materials and methods}

Protein expression, purification and site-directed mutagenesis

Escherichia coli BL21 (DE3) with pET20b plasmid was used as transformant for expression of T. fusca cutinase (residues 1-261) and cutinase- $\mathrm{CBM}_{\mathrm{CenA}}$ (Chen et al. 2008; Zhang et al. 2011), and grown in TB medium supplemented with $0.1 \mathrm{mg} / \mathrm{mL}$ ampicillin at $37^{\circ} \mathrm{C}$ until the $\mathrm{OD}_{600}$ reached $0.6-0.8$, then $16^{\circ} \mathrm{C}$ for $20 \mathrm{~h}$ with $0.1 \mathrm{mM}$ isopropyl $\beta$-D-l-thiogalactopyranoside (IPTG). The cell pellet was harvested by centrifugation at $6000 \mathrm{rpm}$ for $20 \mathrm{~min}$ at $20^{\circ} \mathrm{C}$, and the total protein was precipitated with $70 \%$ saturated ammonium sulfate, collected by centrifugation at $16,000 \mathrm{rpm}$ for $30 \mathrm{~min}$. The pellet was resuspended in $20 \mathrm{~mL}$ Tris buffer (pH 8.0), dialyzed overnight to remove the salt, and purified by nickel affinity chromatography and Superdex 75 10/30 GL column (GE Healthcare, Sweden) gel filtration chromatography. Protein concentration was determined to $10.2 \mathrm{mg} / \mathrm{mL}$, measured by Quantity One electrophoresis gels analyzing (Bio-Rad, USA).

Site-directed mutagenesis was performed by KOD plus Mutagenesis Kit (Toyobo, Japan). Primers used in this work are listed in Additional file 1: Table S1. The parental T. fusca cutinase plasmid was removed by digesting with $D p n \mathrm{I}$ (Biolabs, UK), and PCR products were transformed to DMT chemical competent cells, then amplified to $E$. coli DH5 $\alpha$ cells and BL21 (DE3) for expression. The DNA sequences were sequenced by Biosune Co. LTD., China. Mutations were expressed and purified as WT T. fusca cutinase.

\section{Protein crystallization and X-ray data collection}

The protein was crystallized by mixing $2 \mu \mathrm{L}$ protein (25 mM Tris, $150 \mathrm{mM} \mathrm{NaCl}, \mathrm{pH}$ 8.0) with an equal volume of precipitant (3.0 M NaCl, $4.0 \%(\mathrm{v} / \mathrm{v})$ polypropylene glycol P400, 0.1 M HEPES, pH 7.0), and equilibrated against a $500-\mu \mathrm{L}$ reservoir of the latter. The diffraction quality crystal was grown at $293 \mathrm{~K}$ within a week using hanging drop vapor diffusion method. A data set of $1.54 \AA$ resolution was collected in 2010 at $100 \mathrm{~K}$ with 99.8\% completeness, on Shanghai Synchrotron Radiation Facility beamline 17U1 $(\lambda=0.9796 \AA)$, and processed with the HKL2000 package (Otwinowski and Minor 1997). Using the Phaser program from CCP4 (Winn et al. 2011) suite, the structure was solved by molecular replacement using Streptomyces exfoliates lipase structure (PDB ID, 1JFR) as the search model. The model was built manually in COOT (Emsley et al. 2010), refined with PHENIX (Adams et al. 2010) and CNS (Brunger et al. 1998). The model quality was analyzed by the program MolProbity (Chen et al. 2010b). All structure 
figures were prepared with Pymol (Delano 2012), and the electrostatic surface was calculated by Delphi first. The crystallographic statistics are summarized in Additional file 1: Table S2.

\section{Hydrolyze activity analysis for pNPB}

The pNPB hydrolyze assay was used for tentatively determining activities of cutinase variants (Chen et al. 2008). Reactions were performed by a final concentration of $1 \mathrm{mM}$ pNPB in $25 \mathrm{mM}$ Tris (pH 8.0), initiated by $400 \mathrm{ng} /$ $\mathrm{mL}$ protein, and monitored spectrophotometrically (Infinite F2000 Pro, Tecan, Sweden) at $405 \mathrm{~nm}$. All assays were performed in triplicate.

\section{Potentiometric titration analysis for tomato cutin}

The tomato cutin hydrolyze activity was performed by an automatic titrator (902 Titrando, Metrohm, Switzerland), with $0.5 \mathrm{mg}$ tomato cutin in $1 \mathrm{~mL}$ DMSO, $0.5 \mathrm{~mL}$ protein in $18.5 \mathrm{~mL}$ buffer at $50{ }^{\circ} \mathrm{C}$, and titrated with a volumetric $5 \mathrm{mM} \mathrm{NaOH}$ solution at $\mathrm{pH}$ 8.0. The titration calculated the amount of $\mathrm{NaOH}$ consumed for 30-45 min. Tomato cutin was dissolved in DMSO by ultra-sound for $60 \mathrm{~min}$, and $150 \mathrm{mM} \mathrm{NaCl}$ buffer was preheated at $50{ }^{\circ} \mathrm{C}$ for an hour. All reactions were performed in triplicate.

\section{Degradation of cotton fabrics}

For the enzymatic treatments of cotton fabrics, a pretreatment was performed by boiling the raw fiber for $1 \mathrm{~min}$ and then dried it at $30{ }^{\circ} \mathrm{C}$ for $0.5 \mathrm{~h}$ (Zhang et al. 2010). Then the cotton samples ( $1 \mathrm{~g}$ each), were incubated at $50{ }^{\circ} \mathrm{C}$ with $2.34 \mathrm{mM}$ WT cutinase or mutations $(1 \mathrm{~mL})$, $3000 \mathrm{U}$ pectinase $(1 \mathrm{~mL})$ in $38 \mathrm{~mL} 150 \mathrm{mM} \mathrm{NaOH}, \mathrm{pH}$ 8.0. The treatments were performed in shaking air bath at $200 \mathrm{rpm}$ for $12 \mathrm{~h}$. Samples were boiled for $1 \mathrm{~min}$, washed three times and dried overnight under certain condition. Control assays were performed under the same condition, with cutinase and pectinase boiled for $10 \mathrm{~min}$.

\section{Water absorbency}

After washing and drying, the treated cotton fabrics were tested for wettability using distilled water with a drop size of $0.02 \mathrm{~mL}$ as test liquid. Each fabric was tested in ten different areas and the mean time was calculated (Adams et al. 2010).

\section{Scanning election microscopy (SEM)}

The surface morphologies of samples that were characterized by a JSM-6390LV scanning electron microscope (JEOL, Japan) using $10 \mathrm{k}$ voltage and $3500 \times$ magnification (Zhang et al. 2011). The fabric samples were thoroughly dried and coated with gold before scanning.

\section{Protein structure preparations}

The repaired model of $T$. fusca cutinase was prepared in Schrodinger suite software under OPLS3 force field (Harder et al. 2016) prior to docking. Hydrogen atoms were added to the repaired crystal structures according to the physiological $\mathrm{pH}$ (7.0) with the PROPKA (Sondergaard et al. 2011) tool in Protein Preparation tool in Maestro optimize the hydrogen-bond network. Constrained energy minimizations were conducted on the full-atomic models, with heavy atom coverage to $0.3 \AA$.

\section{Ligand structure preparations}

All ligand structures were built in Schrodinger Maestro software (Schrödinger 2015). The LigPrep module in Schrodinger software was introduced for geometric optimization by using OPLS3 force field (Harder et al. 2016). The ionization state of ligands were calculated with Epik (Greenwood et al. 2010) tool employing Hammett and Taft methods in conjunction with ionization and tautomerization tools (Greenwood et al. 2010).

\section{Protein-ligand docking}

The docking of a ligand to the receptor was performed using Glide (Friesner et al. 2004). Cubic boxes centered on the ligand mass center with a radius $8 \AA$ for all ligands defined the docking binding regions. Flexible ligand docking was executed for all structures. Ten poses per ligand out of 20,000 were included in the post-docking energy minimization. The best scored pose for the ligand was chosen as the initial structure for further study.

\section{Binding energy calculation}

The MM/GBSA calculations had been done in Prime (Sirin et al. 2014). The MM/GBSA energy was calculated using Eq. 1, where $E$ denotes energy and includes terms such as protein-ligand van der Waals contacts, electrostatic interactions, ligand desolvation, and internal strain (ligand and protein) energies, using VSGB2.0 implicit solvent model with the OPLS2005 force field. The solvent entropy is also included in the VSGB2.0 energy model, as it is for other Generalized Born (GB) and Poison-Boltzmann (PB) continuum solvent models.

$$
\Delta G_{\text {bind }}=E_{\text {complex }}-E_{\text {protein }}-E_{\text {ligand }}
$$

\section{Molecular dynamics simulations}

All MD simulations were performed in Gromacs 5.1.4 (Pronk et al. 2013). All amino acid residues of the protein were modeled according to their protonation state at neutral $\mathrm{pH}$. The protein was centered in a water box with a distance of $12 \AA$ away from the protein. The total number of atoms was about 26,678 atoms, $23 \mathrm{Na}^{+}$and $21 \mathrm{Cl}^{-}$ ions, and about 7600 water molecules. CHARMM36m 
(Huang et al. 2017) force field was assigned to the protein, water and ions, while the ligands were treated by CgenFF force field (Vanommeslaeghe et al. 2012). The ligands were submitted to GAUSSIAN 09 program (Frisch et al. 2016) for structure optimization at Hartree-Fock 6-31G* level prior to the generation of force field parameters. All bond lengths of hydrogen atoms in the system were constrained using M-SHAKE (Eric Barth et al. 1995). Van der Waals and short-range electrostatic interactions were cut off at $10 \AA$. The whole system was heated linearly at constant volume (NVT ensemble, 1 bar) from 0 to $310 \mathrm{~K}$ over $400 \mathrm{ps}$. Ten nanoseconds equilibration was performed at constant pressure and temperature (NPT ensemble, $310 \mathrm{~K}, 1$ bar) using the Nose-Hoover coupling scheme with two temperature groups. Long-range electrostatic interactions were computed by particle mesh Ewald (PME) summation. Finally, a 200-ns MD simulation with a time step of $2.0 \mathrm{fs}$ was performed for WT $T$. fusca cutinase, L90A and I213A mutants. The MD simulations results were analyzed in Gromacs (Pronk et al. 2013) and VMD (Humphrey et al. 1996). We calculated the binding energy from molecular mechanics with Poisson-Boltzmann and surface area solvation (MM/PBSA) (Genheden and Ryde 2015). Figures were prepared in PyMOL and Inkscape (Yuan et al. 2016).

\section{Data availability}

The atomic coordinates and structural factors of T. fusca cutinase have been deposited in the Protein Data Bank under accession codes $5 \mathrm{ZOA}$, respectively. The data that supported the findings of this study are available from the corresponding author upon request.

\section{Results and discussion}

\section{Complex structure of $T$. fusca cutinase with cutin mimic}

Seeking to elucidate how T. fusca cutinase binds to cutin, we collected the diffraction data set and determined the 1.54- $\AA$ apo-form crystal structure of $T$. fusca cutinase (Additional file 1: Figs. S1, S2a and Table S2) in 2010 via molecular replacement using the atomic coordinates of Streptomyces exfoliates lipase (PDB ID: 1JFR) as a searching model (Wei et al. 1998). Seven years later, the structure of cutinase 1 from Thermobifida cellulosilytica (PDB ID 5LUI) which showed 99\% sequence identity with $T$. fusca cutinase was published. Structure superposition showed that the two structures are almost same with RMSD of 0.165 except for the C-terminal loop region (residues 245-253) (Additional file 1: Fig. S3) (Ribitsch et al. 2017). Similar to other cutinases, the overall structure of $T$. fusca cutinase exhibits a typical a/ $\beta$ hydrolase fold with center sheet wrapped by 14 loop regions and 8 $\alpha$-helices. The catalytic triad comprises Ser130, Asp176, and His208, all of which are located in loop regions
(Additional file 1: Fig. S2a). The main chain $\mathrm{N}$ atoms of residues Tyr60 and Met131 compose the oxyanion hole in the active site (Additional file 1: Fig. S2b) (Martinez et al. 1994). In this apo-structure, a water molecule establishes a dedicated $\mathrm{H}$-bond network with the oxygen atom of Ser130 (2.5 $\AA$ ), the nitrogen atom of Tyr60 (3.0 $\AA$ ), and the nitrogen atom of Met131 (3.1 $\AA$ ) (Additional file 1: Fig. S2b). Compared to the structure of Fusarium solani pisi cutinase published in 2009, T. fusca cutinase bears a more exposed substrate binding site to accommodate the macromolecules better (Additional file 1: Figs. S4, S5) (Liu et al. 2009).

To get the complex structure of cutinase with cutin, we tried co-crystallization and soaking using the catalytically inactive S130C-H208A variant of the T. fusca cutinase with BHET and BHPT, two cutin mimic oligoesters (Additional file 1: Fig. S6). However, the electron densities of the substrate were invisible in the final structures, which might be due to the low affinity between the substrate and the enzyme. Therefore, we chose to dock oligoester $\mathrm{C}_{24} \mathrm{H}_{42} \mathrm{O}_{8}$, a medium-sized mimic of cutin with four ester bonds were introduced to the alkyl hydroxyl acid group in this molecule (Fig. 1c), into crystal structure of our wild-type (WT) T. fusca cutinase using Glide in Schrodinger software (Friesner et al. 2004). To confirm the stabilities of initial docking results, a 200-ns molecular dynamics (MD) simulation for WT cutinase was performed. We got the modeling complex structure represented by the average pose from the final 10-ns MD simulation.

The modeled $\mathrm{C}_{24} \mathrm{H}_{42} \mathrm{O}_{8}$-cutinase complex structure showed that one end of the long-chain hydrophobic oligoester substrate was inserted into the hydrophobic shallow groove, while the other end was exposed to the bulk environment (Fig. 2a). The second scissile ester bond is located in the active site of cutinase. Additionally, the second carbonyl oxygen of $\mathrm{C}_{24} \mathrm{H}_{42} \mathrm{O}_{8}$ occupies the position of the water molecule which was observed in the active site of apo-structure of T. fusca cutinase. It forms hydrogen bonds with the main chain $\mathrm{N}$ atoms of Y60 and M131 which comprise the oxyanion hole by stabilizing the intermediate state of substrate. The hydroxyl group of S130 was postulated as a nucleophile to the carbonyl carbon atom of second carbonyl in $\mathrm{C}_{24} \mathrm{H}_{42} \mathrm{O}_{8}$ (Fig. 2a). Residues located in the two ends of the groove were exposed to bulk environment. They might prevent the enzyme from getting access to the macromolecular substrate (Fig. 2b).

\section{Catalytic activity analysis of designed $T$. fusca cutinase variants}

Guided by structural analyses, 11 bulky residues were mutated to Ala in an attempt to enhance the substrate 

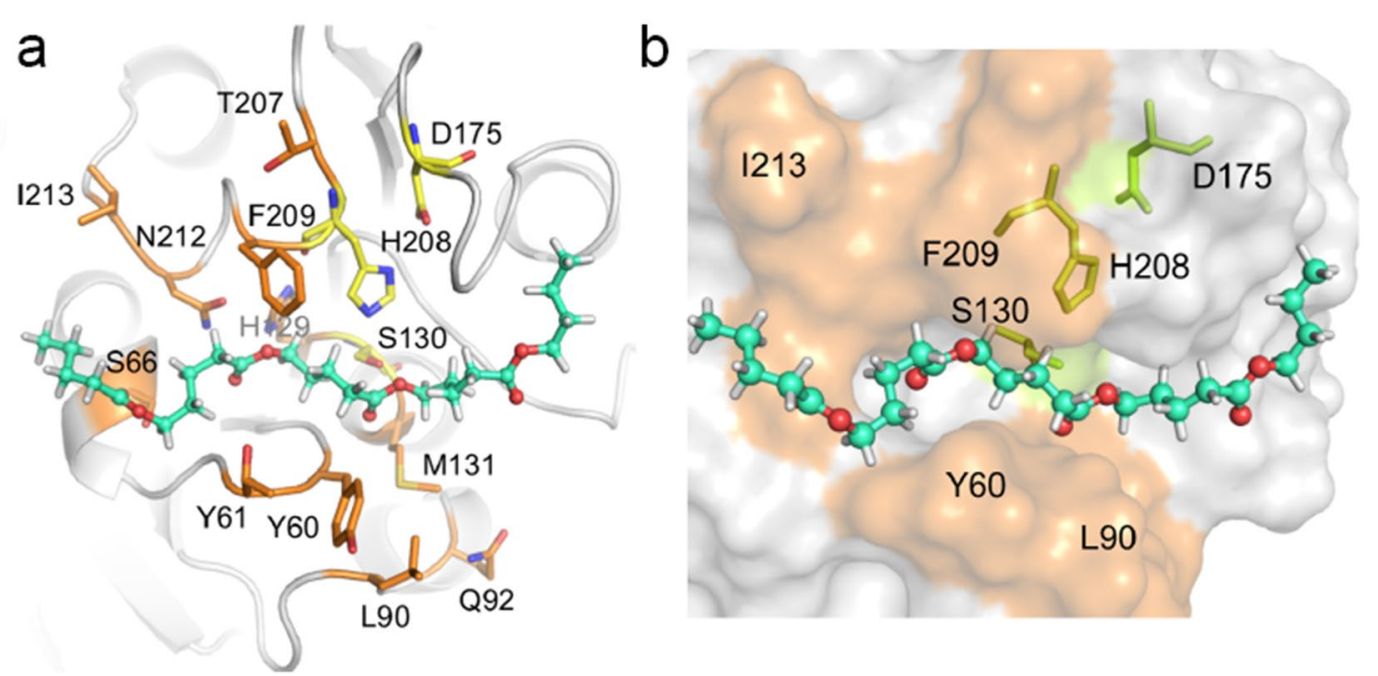

Fig. 2 The structure of a T. fusca cutinase monomer. a Surface and $\mathbf{b}$ cartoon of T. fusca cutinase bound with the oligo-polyester $\mathrm{C}_{24} \mathrm{H}_{48} \mathrm{O}_{8}$ substrate. Green balls-and-sticks: the substrate molecule $\mathrm{C}_{24} \mathrm{H}_{48} \mathrm{O}_{8}$. Yellow sticks: the catalytic residues. Orange sticks: the bulky residues selected to be mutated

binding and hydrolytic activity of $T$. fusca cutinase. Specifically, we designed the variants in two groups (Fig. 2b): the first group (Y60, T61, H129, M131, T207, and F209) includes residues near the catalytic triad and oxygen hole which might preclude S130 from attacking the substrate and/or prevent H208/Y60 from approaching and stabilizing the substrate. The second group of residues (S66, L90, Q92, N212, and I213) are those in the substrate binding site or its vicinity, which were selected in an attempt to increase the enzyme accessibility for long chain or net-sharped substrates.

Firstly, we tested the hydrolysis activities of T. fusca cutinase variants toward $p$-nitrophenyl butyrate $(p N P B)$, a small soluble model substrate of cutinase (Fig. 1b). T61A, H129A, and M131A exhibited significant decreased activity compared with the WT cutinase, suggesting that these residues located near the catalytic triad are not suitable for engineering. Interestingly, two group I variants (Y60A and F209A) and two group II variants (L90A and I213A) showed increasing of $p$ NPB hydrolysis activity (Fig. 3a). Next, to test their catalytic activities toward macromolecules, we carried out enzymatic degradation assay on tomato cuticle cutin, a large insoluble natural polyester substrate. To our surprise, variants L90A, I213A, and L90A/I213A exhibited significant increases in hydrolysis activities toward cutin, 5-, 2.4-, and 3.2-fold higher catalytic activity comparing to the WT cutinase, respectively (Fig. 3b).

\section{Scouring potential analysis of $T$. fusca cutinase variants}

Previous research has shown that the carbohydratebinding modules from Cellulomonas fimi cellulase (CBMcenA) help cutinase bind to the surface of cotton fabrics, which improves scouring efficiency (Zhang et al. 2010, 2011). To test the potential of our enzymes in textile application, we analyzed the efficiencies of selected $T$. fusca cutinase variants (L90A, I213A, and L90A/I213A) compared with WT enzyme in cotton fabrics bioscouring assay. Notably, pectinase is known to confer synergistic effects in this reaction system. Specifically, these assays included a pectinase and variant cutinases which are fused with a CBMcenA domain at the C-terminus. The enzymatic degradation of cuticle of the cotton fabric could increase the fabric wettability, which was used for evaluating the effect of the enzymatic treatment. The results showed that treatment with cutinase will significantly reduce the wetting time of cotton fiber and all three variants exhibited better performances than the WT cutinase, which suggest the stronger hydrolysis ability of variants. Furthermore, cutinase-CBMcenA-treated cotton fiber need less wetting time than the corresponding cutinase (Additional file 1: Fig. S7).

Scouring of cotton fabrics would also cause its morphological changes in surface, which could be analyzed by scanning election microscopy (SEM) (Wang et al. 2006). We scanned the same batches of cotton fabric as that used in the wetting assay. SEM image of the untreated cotton fabrics surface revealed characteristic parallel 


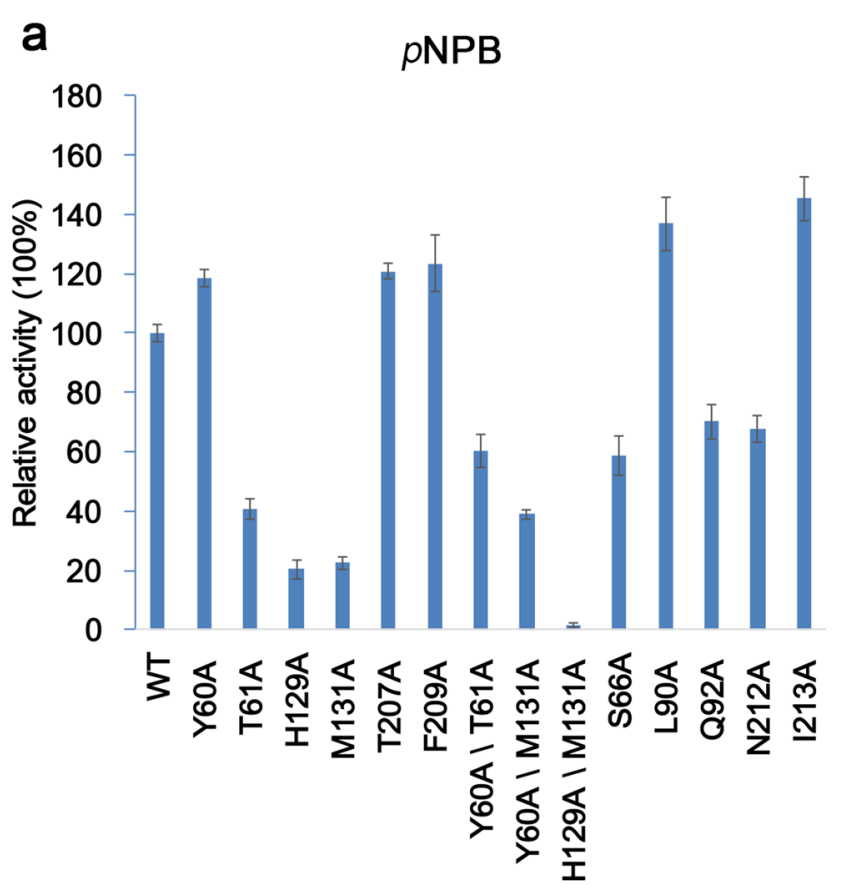

b Tomato cutin

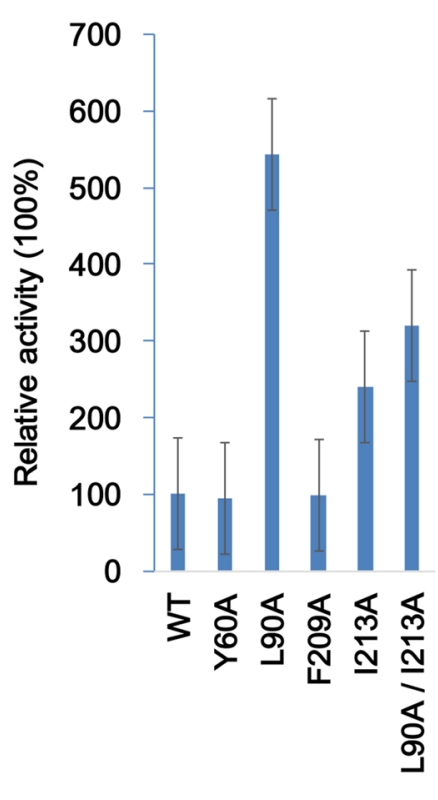

Fig. 3 Hydrolysis activities of the cutinase variants. a Catalytic activities of WT and variant cutinases towards the substrate $p N P B$. $\mathbf{b}$ Hydrolysis activities of the cutinase mu means with standard deviations $(n=3)$

ridges and grooves while alkaline and enzyme-treated fiber presented smoother surface, suggesting a peeling effect of the alkali- or bio-degradation of cutin in the scouring course. Compared to WT-CBMCenA, the surface features of the L90A-CBMCenA, I213A-CBMCenA, and L90A/I213A-CBMCenA-scoured cotton fabrics were clearer and contained less non-cellulosic impurities, indicating that the designed cutinase variants showed higher hydrolysis activities. Moreover, the surface of enzymatic scouring cotton fabrics appeared flatter than the alkaline treated ones bearing layered gully structure, indicating that enzymatic scouring is restricted to the cutin surface. However, the cellulose core is partly destroyed in alkaline condition (Fig. 4).

\section{Structural elucidation of $T$. fusca cutinase variants with enhanced activity}

To explain the enhanced performances of variants L90A and I213A, we modeled the complex structure of the two variants with the substrate mimic $\mathrm{C}_{24} \mathrm{H}_{42} \mathrm{O}_{8}$, using an identical MD simulation protocol as that for WT T. fusca cutinase. We further calculated the mass center distance between the catalytic site and the substrate (Additional file 1: Fig. S8). The MD results show that the substrate mimic is stabilized in the binding pocket all the time which is indicated by movie $1-3$ as well as the RMSD of substrate (Additional file 1: Fig. S9). Free energy calculations revealed that variants L90A and I213A were more favorable than the wild type in substrate recognition: the relative $\Delta G_{\text {bind }}$ were $-10.0 \pm 0.1 \mathrm{kcal} / \mathrm{mol}$, $-14.6 \pm 0.2 \mathrm{kcal} / \mathrm{mol}$, and $-12.4 \pm 0.2 \mathrm{kcal} / \mathrm{mol}$, respectively, for the WT, L90A, and I213A. This data is also supported by the alternation of water-medicated hydrogen-bond network in WT and mutant enzymes. As indicated in Additional file 1: Fig S10, in the WT cutinase, four water-medicated hydrogen-bond networks were observed. However, the corresponding numbers were seven and five, respectively, in variants L90A and I213A. These results are consistent with the data of activity assays in which L90A and I213A showed better catalytic activities towards polyester substrates.

Similar to WT $T$. fusca cutinase, the substrate was located in the groove formed by 4 loop regions, including loop 58-64, loop 84-92, loop 174-182, and loop 204216 in the complex model of variants L90A and I213A (Fig. 5a, b). However, the conformations of the residues in these four loops exhibited diverse orientations in the three forms of the enzyme (Fig. 5a, b). Root-mean-square fluctuations (RMSF) analysis of WT $T$. fusca cutinase and variants without substrate showed that the replacement of bulky residues by alanine not only enhanced the fluctuation of the loop where the mutated residues were located, but also increased the variability of the loops in the vicinity (Fig. 5c). The smaller residues render bigger 

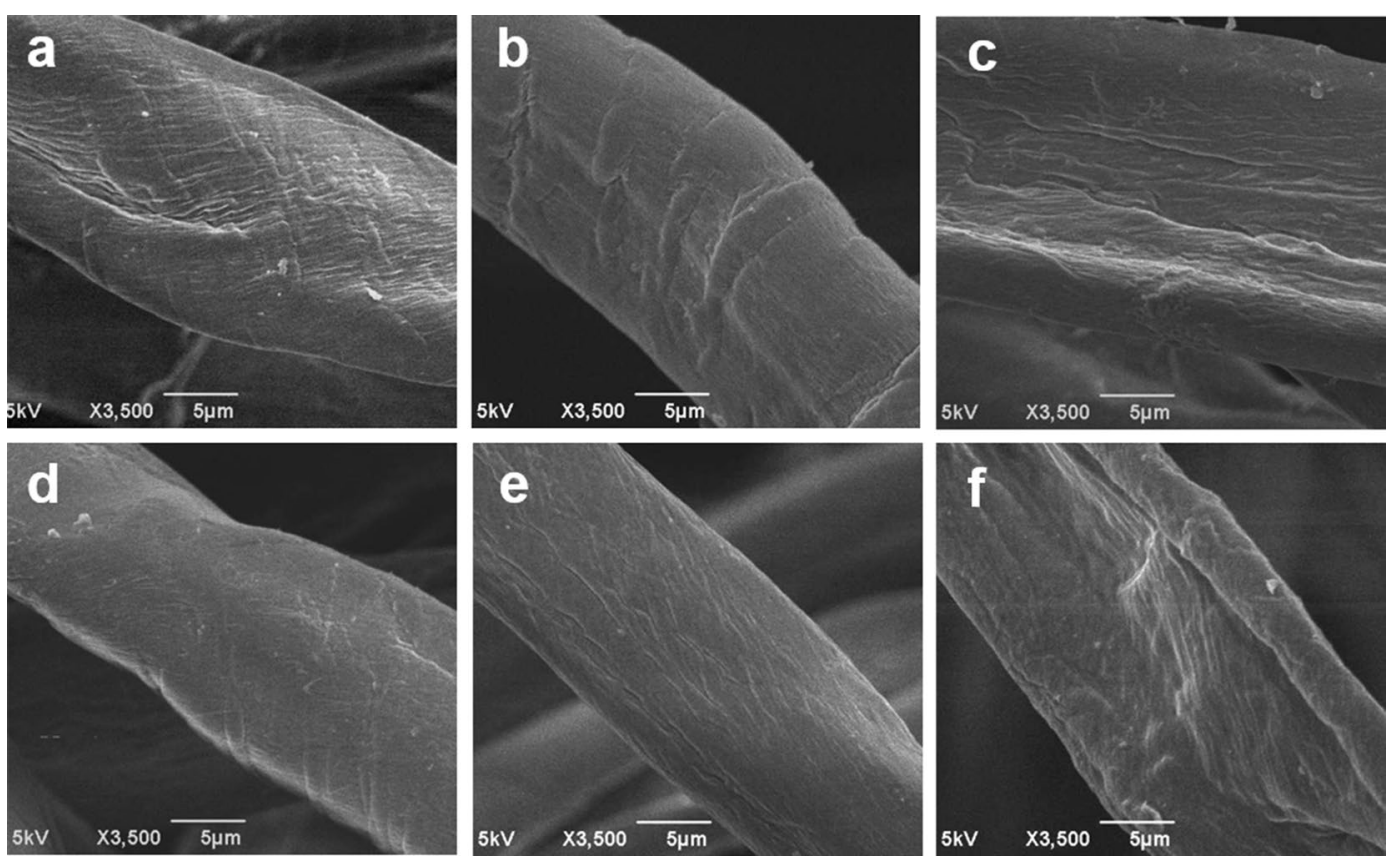

Fig. 4 SEM micrographs of cotton fibers treated with a inactivated cutinase, $\mathbf{b}$ alkaline scouring, c WT-CBMCenA, d L90A-CBMCenA, e I213A-CBMCenA, or $\mathbf{f}$ L90A/I213A CBMCenA. All cutinases were used in combination with pectinase
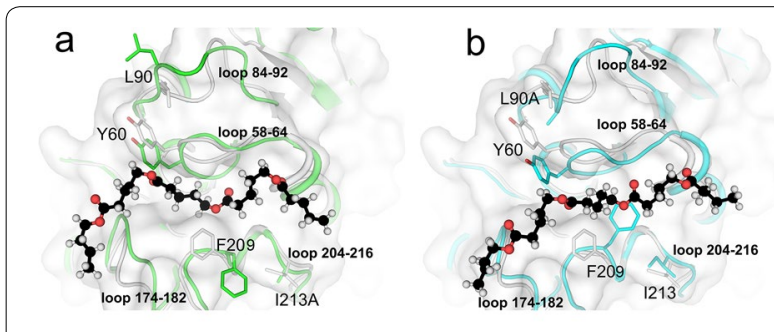

C

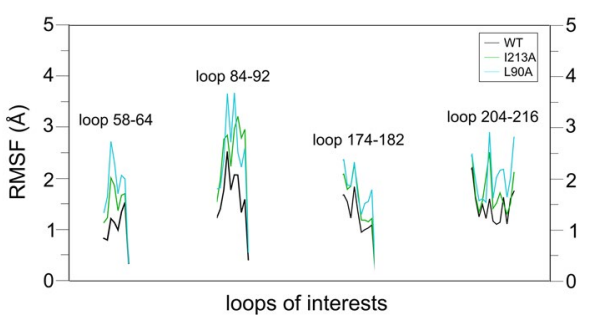

Fig. 5 The conformation of two T. fusca cutinase variants $1213 \mathrm{~A}$ and L90A after MD simulations. The oligoester $\mathrm{C}_{24} \mathrm{H}_{42} \mathrm{O}_{8}$ bound complex structures of $1213 \mathrm{~A}(\mathbf{a})$ and L90A (b) compared with WT. Four loops, including loop 58-64, loop 84-92, loop 174-182 and loop 204-216 are shown as cartoons and sticks in 1213A (green), L90A (cyan) and WT (grey). The substrate $\mathrm{C}_{24} \mathrm{H}_{42} \mathrm{O}_{8}$ is shown as black balls-and-sticks. c The RMSF of these four loops of T. fusca cutinase variants for WT(black), I213A (green) and L90A (cyan) without substrate during the MD simulations space that facilitates the movement of loops surrounding the substrate. Interestingly, the RMSF value of each loop in the simulated complex structures was noticeably higher than that of the ligand-free enzyme, indicating that substrate binding also promotes the fluctuation of four loop regions (Additional file 1: Fig. S11). This may explain why cutinase is able to accommodate and hydrolyze the net-shaped cutin substrate. We also calculated the solvent accessible surface area (SASA) for each case, it is: $2213 \pm 67 \AA 2,2267 \pm 68 \AA 2$ and $2237 \pm 80 \AA 2$ for wild type, L90A and I213A, respectively. It seems that both mutants had a little larger surface (Additional file 1: Fig. S12). Therefore, the more flexible substrate binding site in two L90A and I213A variants might be the key factor to increase hydrolysis activities towards natural cutin substrate.

\section{Conclusion}

Previously, although several engineering efforts have been made to increase the catalytic activity of cutinase on synthetic aromatic polyesters PET, little progress was achieved regarding the cutinase engineering on its natural substrate cutin (Additional file 1: Table S3). In this work, we used a structure-guided protein engineering strategy to improve the hydrolysis ability of a T. fusca cutinase toward cutin and demonstrated the successful use of our improved variants for cotton scouring. The cutinase $-\mathrm{C}_{24} \mathrm{H}_{42} \mathrm{O}_{8}$ modeled complex 
structure which is different from the small substratebound structures would expand the knowledge on polymer substrate binding mode. Replacement of L90 or I213, two bulky residue located in a loop near the substrate binding site-but that do not interact directly with the substrate-increased the flexibility of the adjacent substrate-interacting loop and enlarged the solvent-accessible surface area by increasing the empty space in this region, thus indirectly improving overall cutinase activity. Besides cotton scouring, our successful engineering might be also applied to other polyester degradation, such as PET. In summary, our results illustrate a general strategy of engineering 'indirect' residues which could merit rational protein design when working with enzymes that have exposed substratebinding site and less flexible polymer substrates.

\section{Supplementary information}

Supplementary information accompanies this paper at https://doi. org/10.1186/s40643-020-00324-8.

Additional file 1: Table S1. Primers used in this work. Table S2. Statistics of data collection and structure refinement. Table S3. Engineered mutations of cutinases were reported previously. Fig. S1. Crystal growth of T. fusca cutinase. Fig. S2. Crystal structure of T. fusca cutinase. Fig. S3. Structural comparison of T. fusca cutinase (golden) and cutinase 1 (cyan). The loops at the C-term with different conformation was marked by cycle. Fig. S4. Structural comparison of F. solani pisi cutinase and T. fusca cutinase. Fig. S5. Surface feature comparison of the active site of F. solani pisi cutinase and T. fusca cutinase. Fig. S6. The molecule structures of BHET and BHPT that were used in co-complex crystallization experiments. Fig. S7. Wetting time of cotton fabric after scouring by WT T. fusca cutinase or mutants together with pectinase. Fig. S8. The mass center distances between binding pocket and substrate for WT T. fusca cutinase (black), L90A (cyan) and I213A (green). Fig. S9. The root-mean-square deviation of substrate for WT T. fusca cutinase (black), L90A (cyan) and I213A (green). Fig. S10. The 2D interaction diagram between substrate and (a) WT T. fusca cutinase (b) variant L90A and (c) variant 1213A. Hydrogen bond interactions were indicated by purple arrow. Polar residues were indicated by cyan eclipse. Hydrophobic residues were indicated by green eclipse. The interaction frequency (in percentage) was calculated from MD simulations. Fig. S11. The RMSF of these four loops of T. fusca cutinase variants for WT (black), I213A (green) and L90A (cyan) with substrate during the MD simulations. Fig. S12. The binding pocket solvent accessible surface area (SASA) for WT T. fusca cutinase (black), variant L90A(cyan) and variant I213A(green).

\section{Abbreviations}

PET: Polyethylene terephthalate; WT: Wild type; MD: Molecular dynamics; pNPB: -Nitrophenyl butyrate; SEM: Scanning election microscopy; RMSF: Root-mean-square fluctuations; PME: Particle mesh Ewald; IPTG: Isopropyl- $\beta$-Dthiogalactopyranoside; SASA: Solvent-accessible surface area.

\section{Acknowledgements}

We thank the staff of beamline BL17U1 of Shanghai Synchrotron Radiation Facility (China) for access and help with the X-ray data collection. This work was supported by the Strategic Priority Research Program (B) of the Chinese Academy of Sciences (XDB20000000), Shanghai Municipal Science and Technology Committee (15JC1400403, 19XD1404800) and the National Natural Science Foundation of China (11811530637). The molecular modeling was performed at the Interdisciplinary Centre for Mathematical and Computational Modeling in Warsaw, Poland (GB70-3 and GB71-3).

\section{Authors' contributions}

$\mathrm{QD}, \mathrm{JW}$ and JZ conceived the project. QD and LW carried out the protein purification, determined the crystal structure, performed biochemical assays and SEM assays. SY performed the molecular modeling and molecular dynamics simulations. QZ helped the SEM assays. LS helped preparing cell pellets and cotton fibers. QD, SY, WH and JZ interpreted the data and wrote the manuscript. All authors read and approved the final manuscript.

\section{Funding}

This work was supported by the Strategic Priority Research Program (B) of the Chinese Academy of Sciences (XDB20000000), Shanghai Municipal Science and Technology Committee (15JC1400403, 19XD1404800) and the National Natural Science Foundation of China (11811530637).

\section{Availability of data and materials}

The data set (table and graphs) supporting this article's conclusion is available.

Ethics approval and consent to participate

Not applicable.

\section{Consent for publication}

Not applicable.

\section{Competing interests}

The authors declare that they have no competing interests.

\section{Author details}

${ }_{1}^{1}$ State Key Laboratory of Bioorganic and Natural Products Chemistry, Center for Excellence in Molecular Synthesis, Shanghai Institute of Organic Chemistry, Chinese Academy of Sciences, 345 Lingling Road, Shanghai 200032, China. ${ }^{2}$ Shaanxi Key Laboratory of Natural Products \& Chemical Biology, College of Chemistry and Pharmacy, Northwest A\&F University, 3 Taicheng Road, Yangling 712100, Shaanxi, China. ${ }^{3}$ State Key Laboratory of Medical Chemical Biology, Nankai University, Tianjin 300071, China. ${ }^{4}$ Shenzhen Institutes of Advanced Technology, Chinese Academy of Sciences, Shenzhen 518055 , China. ${ }^{5}$ State Key Laboratory of Food Science and Technology, Jiangnan University, 1800 Lihu Avenue, Wuxi 214122, China.

Received: 19 February 2020 Accepted: 16 June 2020

Published online: 09 July 2020

\section{References}

Adams PD, Afonine PV, Bunkoczi G, Chen VB, Davis IW, Echols N, Headd JJ, Hung LW, Kapral GJ, Grosse-Kunstleve RW, McCoy AJ, Moriarty NW, Oeffner R, Read RJ, Richardson DC, Richardson JS, Terwilliger TC, Zwart PH (2010) PHENIX: a comprehensive Python-based system for macromolecular structure solution. Acta Crystallogr D Biol Crystallogr 66(Pt 2):213-221

Araujo R, Silva C, O'Neill A, Micaelo N, Guebitz G, Soares CM, Casal M, CavacoPaulo A (2007) Tailoring cutinase activity towards polyethylene terephthalate and polyamide 6,6 fibers. J Biotechnol 128(4):849-857

Austin HP, Allen MD, Donohoe BS, Rorrer NA, Kearns FL, Silveira RL, Pollard BC, Dominick G, Duman R, El Omari K, Mykhaylyk V, Wagner A, Michener WE, Amore A, Skaf MS, Crowley MF, Thorne AW, Johnson CW, Woodcock HL, McGeehan JE, Beckham GT (2018) Characterization and engineering of a plastic-degrading aromatic polyesterase. Proc Natl Acad Sci USA 115(19):E4350-E4357

Brunger AT, Adams PD, Clore GM, DeLano WL, Gros P, Grosse-Kunstleve RW, Jiang JS, Kuszewski J, Nilges M, Pannu NS, Read RJ, Rice LM, Simonson T, Warren GL (1998) Crystallography \& NMR system: a new software suite for macromolecular structure determination. Acta Crystallogr D Biol Crystallogr 54(Pt 5):905-921

Chen S, Tong X, Woodard RW, Du G, Wu J, Chen J (2008) Identification and characterization of bacterial cutinase. J Biol Chem 283(38):25854-25862

Chen S, Su L, Billig S, Zimmermann W, Chen J, Wu J (2010a) Biochemical characterization of the cutinases from Thermobifida fusca. J Mol Catal B Enzym 63(3-4):121-127

Chen VB, Arendall WB 3rd, Headd JJ, Keedy DA, Immormino RM, Kapral GJ, Murray LW, Richardson JS, Richardson DC (2010b) MolProbity: all-atom 
structure validation for macromolecular crystallography. Acta Crystallogr D Biol Crystallogr 66(Pt 1):12-21

Delano WL (2012) The PyMOL molecular graphics system, Version 1.50

Emsley P, Lohkamp B, Scott WG, Cowtan K (2010) Features and development of coot. Acta Crystallogr D Biol Crystallogr 66:16

Eric Barth KK, Leimkuhler Benedict, Skeel Robert D (1995) Algorithms for constrained molecular dynamics. J Comput Chem 16(10):18

Fecker T, Galaz-Davison P, Engelberger F, Narui Y, Sotomayor M, Parra LP, Ramirez-Sarmiento CA (2018) Active site flexibility as a hallmark for efficient PET degradation by I. sakaiensis PETase. Biophys J 114(6):1302-1312

Friesner RA, Banks JL, Murphy RB, Halgren TA, Klicic JJ, Mainz DT, Repasky MP, Knoll EH, Shelley M, Perry JK, Shaw DE, Francis P, Shenkin PS (2004) Glide: a new approach for rapid, accurate docking and scoring. 1. Method and assessment of docking accuracy. J Med Chem 47(7):1739-1749

Frisch MJ, Trucks GW, Schlegel HB, Scuseria GE, Robb MA, Cheeseman JR, Scalmani G, Barone V, Petersson GA, Nakatsuji H, Li X, Caricato M, Marenich A, Bloino J, Janesko BG, Gomperts R, Mennucci B, Hratchian HP, Ortiz JV, Izmaylov AF, Sonnenberg JL, Williams-Young D, Ding F, Lipparini F, Egidi F, Goings J, Peng B, Petrone A, Henderson T, Ranasinghe D, Zakrzewski VG, Gao J, Rega N, Zheng G, Liang W, Hada M, Ehara M, Toyota K, Fukuda R, Hasegawa J, Ishida M, Nakajima T, Honda Y, Kitao O, Nakai H, Vreven T, Throssell K, Montgomery JA Jr, Peralta JE, Ogliaro F, Bearpark M, Heyd JJ, Brothers E, Kudin KN, Staroverov VN, Keith T, Kobayashi R, Normand J, Raghavachari K, Rendell A, Burant JC, lyengar SS, Tomasi J, Cossi M, Millam JM, Klene M, Adamo C, Cammi R, Ochterski JW, Martin RL, Morokuma K, Farkas O, Foresman JB, Fox DJ (2016) Revision A.02. Gaussian Inc, Wallingford CT

Genheden S, Ryde U (2015) The MM/PBSA and MM/GBSA methods to estimate ligand-binding affinities. Expert Opin Drug Discov 10(5):449-461

Greenwood JR, Calkins D, Sullivan AP, Shelley JC (2010) Towards the comprehensive, rapid, and accurate prediction of the favorable tautomeric states of drug-like molecules in aqueous solution. J Comput Aided Mol Des 24(6-7):591-604

Han X, Liu W, Huang JW, Ma J, Zheng Y, Ko TP, Xu L, Cheng YS, Chen CC, Guo RT (2017) Structural insight into catalytic mechanism of PET hydrolase. Nat Commun 8(1):2106

Harder E, Damm W, Maple J, Wu C, Reboul M, Xiang JY, Wang L, Lupyan D, Dahlgren MK, Knight JL, Kaus JW, Cerutti DS, Krilov G, Jorgensen WL, Abel R, Friesner RA (2016) OPLS3: a force field providing broad coverage of drug-like small molecules and proteins. J Chem Theory Comput 12(1):281-296

Hegde K, Dasu WV (2014) Structural stability and unfolding properties of cutinases from Thermobifida fusca. Appl Biochem Biotechnol 174(2):803-819

Huang J, Rauscher S, Nawrocki G, Ran T, Feig M, de Groot BL, Grubmuller H, MacKerell AD Jr (2017) CHARMM36m: an improved force field for folded and intrinsically disordered proteins. Nat Methods 14(1):71-73

Humphrey W, Dalke A, Schulten K (1996). VMD: visual molecular dynamics. J Mol Graph 14(1), 33-38, 27-38

Joo S, Cho IJ, Seo H, Son HF, Sagong HY, Shin TJ, Choi SY, Lee SY, Kim KJ (2018) Structural insight into molecular mechanism of poly(ethylene terephthalate) degradation. Nat Commun 9(1):382

Kitadokoro K, Thumarat U, Nakamura R, Nishimura K, Karatani H, Suzuki H, Kawai F (2012) Crystal structure of cutinase Est119 from Thermobifida alba AHK119 that can degrade modified polyethylene terephthalate at $1.76 \AA$ resolution. Polym Degrad Stabil 97(5):771-775

Kolattukudy PE (2001) Polyesters in higher plants. Adv Biochem Eng Biotechnol 71:1-49

Liu Z, Gosser Y, Baker PJ, Ravee Y, Lu Z, Alemu G, Li H, Butterfoss GL, Kong XP, Gross R, Montclare JK (2009) Structural and functional studies of Aspergillus oryzae cutinase: enhanced thermostability and hydrolytic activity of synthetic ester and polyester degradation. J Am Chem Soc 131(43):15711-15716

Martinez C, Nicolas A, van Tilbeurgh H, Egloff MP, Cudrey C, Verger R, Cambillau C (1994) Cutinase, a lipolytic enzyme with a preformed oxyanion hole. Biochemistry 33(1):83-89

Nyyssola A (2015) Which properties of cutinases are important for applications? Appl Microbiol Biotechnol 99(12):4931-4942

Oda M, Yamagami Y, Inaba S, Oida T, Yamamoto M, Kitajima S, Kawai F (2018) Enzymatic hydrolysis of PET: functional roles of three $\mathrm{Ca}(2+)$ ions bound to a cutinase-like enzyme, Cut $190^{*}$, and its engineering for improved activity. Appl Microbiol Biotechnol 102(23):10067-10077
Otwinowski Z, Minor W (1997) Processing of X-ray diffraction data collected in oscillation mode. Methods Enzymol 276:307-326

Pronk S, Pall S, Schulz R, Larsson P, Bjelkmar P, Apostolov R, Shirts MR, Smith JC, Kasson PM, van der Spoel D, Hess B, Lindahl E (2013) GROMACS 4.5: a high-throughput and highly parallel open source molecular simulation toolkit. Bioinformatics 29(7):845-854

Purdy RE, Kolattukudy PE (1973) Depolymerization of a hydroxy fatty acid biopolymer, cutin, by an extracellular enzyme from Fusarium solani $\mathrm{f}$. pisi: isolation and some properties of the enzyme. Arch Biochem Biophys 159(1):61-69

Purdy RE, Kolattukudy PE (1975) Hydrolysis of plant cuticle by plant pathogens. Properties of cutinase I, cutinase II, and a nonspecific esterase isolated from Fusarium solani pisi. Biochemistry 14(13):2832-2840

Ribitsch D, Acero EH, Greimel K, Eiteljoerg I, Trotscha E, Freddi G, Schwab H, Guebitz GM (2011) Characterization of a new cutinase from Thermobifida alba for PET-surface hydrolysis. Biocatal Biotransform 30(1):2-9

Ribitsch D, Altijana HA, Zitzenbacher S, Zartl B, Gamerith C, Pellis A, Jungbauer A, Łyskowski A, Steinkellner G, Gruber K, Tscheliessnig R, Acero EH, Guebitz GM (2017) Small cause, large effect: structural characterization of cutinases from Thermobifida cellulosilytica. Biotechnol Bioeng 114(11):2481-2488

Saavedra JM, Azocar MA, Rodriguez V, Ramirez-Sarmiento CA, Andrews BA, Asenjo JA, Parra LP (2018) Relevance of local flexibility near the active site for enzymatic catalysis: biochemical characterization and engineering of cellulase Cel5A from Bacillus agaradherans. Biotechnol J 13(8):e1700669

Schrödinger LS, LLC (2015) Maestro. Schrödinger, LLC, New York

Silva C, Da S, Silva N, Matama T, Araujo R, Martins M, Chen S, Chen J, Wu J, Casal M, Cavaco-Paulo A (2011) Engineered Thermobifida fusca cutinase with increased activity on polyester substrates. Biotechnol J 6(10):1230-1239

Sirin S, Kumar R, Martinez C, Karmilowicz MJ, Ghosh P, Abramov YA, Martin V, Sherman W (2014) A computational approach to enzyme design: predicting omega-aminotransferase catalytic activity using docking and MM-GBSA scoring. J Chem Inf Model 54(8):2334-2346

Sondergaard CR, Olsson MH, Rostkowski M, Jensen JH (2011) Improved treatment of ligands and coupling effects in empirical calculation and rationalization of pKa values. J Chem Theory Comput 7(7):2284-2295

Tournier V, Topham CM, Gilles A, David B, Folgoas C, Moya-Leclair E, Kamionka E, Desrousseaux ML, Texier H, Gavalda S, Cot M, Guemard E, Dalibey M, Nomme J, Cioci G, Barbe S, Chateau M, Andre I, Duquesne S, Marty A (2020) An engineered PET depolymerase to break down and recycle plastic bottles. Nature 580(7802):216-219

Vanommeslaeghe K, Raman EP, MacKerell AD Jr (2012) Automation of the CHARMM General Force Field (CGenFF) II: assignment of bonded parameters and partial atomic charges. J Chem Inf Model 52(12):3155-3168

Wang Q, Fan X, Gao W, Chen J (2006) Characterization of bioscoured cotton fabrics using FT-IR ATR spectroscopy and microscopy techniques. Carbohydr Res 341(12):2170-2175

Wei Y, Swenson L, Castro C, Derewenda U, Minor W, Arai H, Aoki J, Inoue K, Servin-Gonzalez L, Derewenda ZS (1998) Structure of a microbial homologue of mammalian platelet-activating factor acetylhydrolases: Streptomyces exfoliatus lipase at 1.9 A resolution. Structure 6(4):511-519

Winn MD, Ballard CC, Cowtan KD, Dodson EJ, Emsley P, Evans PR, Keegan RM, Krissinel EB, Leslie AG, McCoy A, McNicholas SJ, Murshudov GN, Pannu NS, Potterton EA, Powell HR, Read RJ, Vagin A, Wilson KS (2011) Overview of the CCP4 suite and current developments. Acta Crystallogr D Biol Crystallogr 67(Pt 4):235-242

Yuan S, Chan HCS, Filipek S, Vogel H (2016) PyMOL and inkscape bridge the data and the data visualization. Structure 24(12):2041-2042

Zhang Y, Chen S, Xu M, Cavaco-Paulo A, Wu J, Chen J (2010) Characterization of Thermobifida fusca cutinase-carbohydrate-binding module fusion proteins and their potential application in bioscouring. Appl Environ Microbiol 76(20):6870-6876

Zhang Y, Chen S, He M, Wu J, Chen J, Wang Q (2011) Effects of Thermobifida fusca cutinase-carbohydrate-binding module fusion proteins on cotton bioscouring. Biotechnol Bioprocess Eng 16(4):645-653

\section{Publisher's Note}

Springer Nature remains neutral with regard to jurisdictional claims in published maps and institutional affiliations. 